\title{
A REMARK ABOU'T A CERTAIN CLASS OF DISTRIBUTION SPACES
}

\author{
S. R. HARASYMIV \\ (Received 19 August 1968) \\ Communicated by J. B. Miller
}

\section{Introduction}

The object of this note is to exhibit a certain class of distribution spaces as being $c$-admissible in the sense of [1] and [2]. Throughout the terminology and notation are the same as in [1] and [2]. The only addition to this is that if $x \in R^{n}$ then $\tau_{x}$ will denote the translation operator which carries each distribution $u$ onto the distribution $u_{x}$.

\section{2. c-admissibility of certain spaces}

We shall prove the following result.

Proposition. Let $\boldsymbol{E}$ be an admissible space which is barelled and $B_{r^{-}}$ complete, and which is a module over $\boldsymbol{S}$ with respect to convolution.

Consider the following two hypotheses:

(i) $\boldsymbol{E}$ is translation-invariant and for each $u \in \boldsymbol{E}$ the mapping $\boldsymbol{x} \rightarrow u_{x}$ of $R^{n}$ into $E$ is bounded on compact subsets of $R^{n}$.

(ii) $\boldsymbol{E}$ is dilation-invariant and for each $u \in \boldsymbol{E}$ the mapping $x \rightarrow u^{x}$ of $R^{\#}$ into $\boldsymbol{E}$ is bounded on compact subsets of $R^{\#}$

Then the conclusions are:

(a) If (i) holds then $\boldsymbol{E}$ is c-admissible.

(b) If both (i) and (ii) hold then $\boldsymbol{E}$ is a dilation space.

Proof. We shall begin with the proof of assertion (a). Thus we assume that (i) holds.

Our first task is to show that for each $x \in R^{n}$ the mapping $u \rightarrow u_{x}$ of $\boldsymbol{E}$ into itself is continuous. Since $\boldsymbol{E}$ is both barrelled and $B_{r}$-complete, the closed graph theorem (Theorem 8.9.4 in Edwards [3] and the first Remark following it) tells us that it is sufficient to show that the linear operator $\tau_{x}$ (considered as a mapping of $\boldsymbol{E}$ into itself) has a closed graph. To this end 
we assume that $x \in R^{n}$ is fixed, and that $\left(u_{i}\right)$ is a net in $E$ such that $\lim _{i} u_{i}=u$ in $E$ and $\lim \tau_{x} u_{i}=w$ in $\boldsymbol{E}$. Then for each $\phi \in S$ it follows (because of relation (2.1) in [1]) that

$$
\begin{aligned}
w * \phi(0) & =\lim _{i}\left(\tau_{x} u_{i}\right) * \phi(0) \\
& =\lim _{i} u_{i} * \phi_{x}(0) \\
& =u * \phi_{x}(0) \\
& =\left(\tau_{x} u\right) * \phi(0)
\end{aligned}
$$

Thus $w=\tau_{x} u$ and the graph of $\tau_{x}$ is indeed closed.

Our second requirement is to show that for each $u \in E$ and each $v \in E^{\prime}$ the mapping $x \rightarrow\left\langle u_{x}, v\right\rangle$ defines a continuous function on $R^{n}$. With this end in mind, let $b \in R^{n}$ be arbitrary but fixed. Let $K$ be the set $\left\{x \in R^{n}:|x-b| \leqq 1\right\}$ and consider the set $\left\{\tau_{x}: x \in K\right\}$ of continuous linear mappings of $\boldsymbol{E}$ into itself. Write $\boldsymbol{E}_{\mathbf{0}}$ for the set of all $u \in \boldsymbol{E}$ for which $\lim _{x \rightarrow b} \tau_{x} u$ exists in $\boldsymbol{E}$ and define the mapping $T$ of $\boldsymbol{E}_{0}$ into $\boldsymbol{E}$ by $T u=\lim _{x \rightarrow b} \tau_{x x} u\left(u \in E_{0}\right)$. We notice that for each fixed $\phi \in S$, the mapping $x \in R^{n} \rightarrow \phi_{x} \in \boldsymbol{S} \rightarrow \phi_{x} \in \boldsymbol{E}$ is continuous; and hence that

$$
\lim _{x \rightarrow b} \tau_{x} \phi=\tau_{b} \phi \text { in } E .
$$

It follows from this that $\boldsymbol{E}_{0}$ contains $\boldsymbol{S}$, which is dense in $\boldsymbol{E}$. Secondly, our assumption that (i) holds entails that the set $\left\{\tau_{x}: x \in K\right\}$ of continuous linear mappings is bounded at each point of $\boldsymbol{E}$. Thus, since $\boldsymbol{E}$ is $B_{\tau}$-complete and hence quasi-complete, we may refer to Corollary 7.1.4 in Edwards [3] and deduce that $\boldsymbol{E}_{\mathbf{0}}=\boldsymbol{E}$ and that $T$ is a continuous linear mapping of $\boldsymbol{E}$ into itself. But relation (2.1) shows that $T$ coincides with the continuous linear mapping $\tau_{b}$ on the dense vector subspace $\boldsymbol{S}$ of $\boldsymbol{E}$; whence it follows that the two mappings are identical. Thus $\lim _{x \rightarrow b} \tau_{x} u=\tau_{b} u$ for each $u \in E$. Since $b \in R^{n}$ is arbitrary, we now infer that for each $u \in E$, the mapping $x \rightarrow u_{x}$ is continuous from $R^{n}$ into $\boldsymbol{E}$. It follows immediately that for each $u \in \boldsymbol{E}$ and each $v \in \boldsymbol{E}^{\prime}$, the mapping $x \rightarrow\left\langle u_{x}, v\right\rangle$ defines a continuous function on $R^{n}$, which is what we wished to prove.

Next consider a fixed $\phi \in \boldsymbol{S}$. We claim that the mapping $u \rightarrow u * \phi$ of $\boldsymbol{E}$ into itself is continuous. To verify this assertion, it is sufficient to show that the graph of this mapping is closed; the desired conclusion will then follow from the closed graph theorem. Thus let $\left(u_{i}\right)$ be a net in $\boldsymbol{E}$ such that $\lim _{i} u_{i}=w$ in $E$ and $\lim _{i} u_{i} * \phi=w$ in $E$. Then for each $\psi \in S$ we have $w * \psi(0)=\lim _{i} u_{i} * \phi * \psi(0)=u * \phi * \psi(0)$. Hence $w=u * \phi$ and the mapping $u \in \boldsymbol{E} \rightarrow u * \phi \in \boldsymbol{E}$ is closed, as required.

If we recall that $S$ is barrelled, then a similar argument shows that for each $u \in \boldsymbol{E}$, the mapping $\phi \rightarrow u * \phi$ of $\boldsymbol{S}$ into $\boldsymbol{E}$ is continuous. 
We shall now complete the proof of part (a) of the Proposition. Let $u \in \boldsymbol{E}$ and $v \in \boldsymbol{E}^{\prime}$ be arbitrary but fixed. We must show that the continuous function $x \rightarrow\left\langle u_{x}, v\right\rangle\left(x \in R^{n}\right)$ generates a temperate distribution on $R^{n}$. In view of the last paragraph, the mapping $\phi \rightarrow\langle u * \phi, v\rangle(\phi \in \boldsymbol{S})$ defines a temperate distribution, which we denote by $s$. We shall show that the function $x \rightarrow\left\langle u_{x}, v\right\rangle\left(x \in R^{n}\right)$ generates precisely this distribution $s$. To do this it is sufficient to show that for each $\psi \in \boldsymbol{D}$

$$
\int_{R^{n}}\left\langle u_{x}, v\right\rangle \psi(-x) d x=s * \psi(0) .
$$

Let $\psi \in \boldsymbol{D}$ be arbitrary. Choose a. net $\left(\phi_{i}\right)$ in $\boldsymbol{S}$ such that $\lim _{i} \phi_{i}=u$ in $\boldsymbol{E}$. We notice that, because of Theorem $2.2(a)$ in [1] and the continuity of the functions $x \rightarrow\left\langle u_{x}, v\right\rangle$ on $R^{n}$, the mapping $x \rightarrow v_{x}$ is continuous from $R^{n}$ into $\boldsymbol{E}^{\prime}$ for the weak topology on $\boldsymbol{E}^{\prime}$. Therefore the set $\left\{v_{x}: x \in \operatorname{supp} \psi\right\}$ is a weakly compact, hence weakly bounded. hence equicontinuous (because $\boldsymbol{E}$ is barrelled) subset of $\boldsymbol{E}^{\prime}$. In view of this we conclinde that

$$
\lim _{i} \phi_{i} * v(x)=\lim _{i}\left\langle\phi_{i}, v_{x}\right\rangle=\left\langle u, v_{x}\right\rangle=\left\langle u_{x}, v\right\rangle
$$

uniformly for $x \in \operatorname{supp} \psi$. It follows that

$$
\begin{aligned}
\int_{R^{n}}\left\langle u_{x}, v\right\rangle \psi(-x) d x & =\lim _{i} \int_{R^{n}} \phi_{i} * v(x) \psi(-x) d x \\
& =\lim _{i} \phi_{i} * \psi * v(0) \\
& =\lim _{i}\left\langle\phi_{i} * \psi, v\right\rangle .
\end{aligned}
$$

Now we have shown above that the mapping $w \in \boldsymbol{E} \rightarrow w * \psi \in \boldsymbol{E}$ is continuous. Therefore

$$
\lim _{i}\left\langle\phi_{i} * \psi, v\right\rangle=\langle u * \psi, v\rangle=s * \psi(0) .
$$

Relations (2.3) and (2.4) together ensure that (2.2) holds; whence we infer that the function $x \rightarrow\left\langle u_{x}, v\right\rangle$ can indeed be identified with a temperate distribution on $R^{n}$. Since $u \in \boldsymbol{E}$ and $v \in \boldsymbol{E}^{\prime}$ were arbitrary, this completes the proof of (a).

The validity of part (b) of the Proposition will be established if we can show that (ii) entails that the mapping $x \rightarrow u^{x}$ of $R^{\#}$ into $E$ and the mapping $u \rightarrow u^{x}$ of $E$ into itself are both continuous (for the given topology on $\boldsymbol{E}$ ); and the truth of this may be verified by using arguments analogous to those which we employed above to establish the continuity of the mappings $x \rightarrow u_{x}$ of $R^{n}$ into $E$ and $u \rightarrow u_{x}$ of $\boldsymbol{E}$ into itself.

\section{References}

[1] S. R. Harasymiv, 'On Approximation by dilations of distributions', Pacific $J$. Math. 28 (1969), 363-374. 
[2] S. R. Harasymiv, 'A note on approximation of distributions by quasi-analytic functions', J. Aust. Math. Soc. 10 (1969), 95-105.

[3] R. E. Edwards, Functional Analysis: Theory and Applications (Holt, Rinehart and Winston, New York, 1965).

Department of Mathematics

University of Connecticut 